\title{
Deprescribing: routine pharmacy practice or an exciting research opportunity?
}

\author{
David John Wright, Sion Scott and Debi Bhattacharya \\ School of Pharmacy, University of East Anglia, Norwich, UK
}

doi: 10.1111/ijpp.12513

Every few years a new word becomes prominent in the pharmacy lexicon and without a doubt 'deprescribing' is currently 'à la mode'. The response from pharmacists is generally that deprescribing is central to everything that we do and why do we need a new term for medicines discontinuation? However, deprescribing is 'the systematic process of identifying and discontinuing drugs in instances where existing or potential harms outweigh existing or potential benefits within the context of an individual patient's care goals, current level of functioning, life expectancy, values and preferences'. ${ }^{[1]}$ The important element of this sentence is the differentiation of 'existing harms' from 'potential harms'. We agree that central to the pharmacist's role is reviewing medicines to identify those which require deprescribing. But this is usually in response to a clinical trigger, such as an adverse drug reaction. ${ }^{[2]}$ This deprescribing is termed 'reactive' and is relatively straightforward as it is both unethical and negligent to continue the medication.

We recently reviewed medicine discontinuations within the hospital setting and found that less than $1 \%$ of admission medications are deprescribed. The problem with this statistic is twofold. Firstly, around $50 \%$ of prescribed medication is potentially inappropriate ${ }^{[3]}$ and so the current $1 \%$ of deprescribing activity is clearly not adequately addressing the problem of potentially inappropriate prescribed medication. Secondly, the deprescribing that we observed was overwhelmingly reactive. ${ }^{[2]}$ Proactive deprescribing is where the decision regarding continuation or cessation of therapy is less 'black and white'; the medicine may or may not be effective, and the risk of harm in that patient may no longer be outweighed by the benefits. ${ }^{[2]}$ Proactive deprescribing is undoubtedly more difficult for practitioners to execute than reactive. This is reflected in limited proactive deprescribing being observed in both the secondary care ${ }^{[2]}$ and primary care settings. ${ }^{[4]}$

Systematic reviews of patient and prescriber barriers to and enablers of deprescribing ${ }^{[5]}$ provide insight into current research gaps and the potential role of the pharmacist. An enabler of deprescribing that is relevant to the pharmacy profession is patients' reported preference for, and greater willingness to agree, if the deprescribing recommendation comes from their doctor. ${ }^{[5]}$ This information, however, results from qualitative research where patients were asked open questions regarding the concept. Willingness to stop medication at the recommendation of the pharmacist is unknown, and this may differ depending on context and role, for example pharmacists employed within medical practices may receive different responses to those employed by supporting bodies with a remit of optimizing prescribing. Similarly, pharmacists with and without prescribing rights may have differing responses. Answering this question is important in identifying the potential role of the pharmacist within the practice of proactive deprescribing.

A further enabler to patients engaging with deprescribing is when it is offered as a trial rather than a fait accompli. Furthermore, provision for discussing any problems if they arise postdeprescribing, and restarting medication if necessary, supports discontinuation. This postdiscontinuation activity affords an ideal role for pharmacists either located within the medical practice or community pharmacy plus associated research to develop and test either model.

Evidence regarding prescribers' influencers of deprescribing is dominated by primary care doctors'. ${ }^{[6]}$ The views of pharmacists towards deprescribing remain poorly understood despite the majority of deprescribing intervention trials either being supported or led by pharmacists. $^{[7,8]}$ A significant prescriber barrier to deprescribing is lack of knowledge about how to safely implement it and concerns regarding harming the patient-prescriber relationship plus possible litigation resulting from iatrogenic harms. Whilst the dangers of deprescribing are likely to be no greater than prescribing, the body of evidence supporting deprescribing is markedly smaller. When considering summaries of product characteristics and national prescribing guidance, they clearly state when a medicine should be started, when this should be done with caution and when it should not be done. Furthermore, guidance on dosage tapering, side effects and counselling points is provided as standard. No comparable 
guidance is available for deprescribing, and it is unsurprising therefore that prescribers are concerned about the potential for negative repercussions. Information regarding when to deprescribe, when to consider deprescribing with caution and when deprescribing should not be trialled would be helpful, as would information on dose tapering, side effects and deprescribing counselling points at individual drug level. Guidelines on how to stop certain groups of medication such as proton pump inhibitors $^{[9]}$ and antihyperglycaemic agents ${ }^{[10]}$ are available; however, the detail at individual drug level is unavailable and the information is not located within the reference sources routinely used by healthcare professionals. For obvious reasons, it may not be appropriate for regulatory authorities to mandate manufacturers to provide this information, and therefore, a research opportunity exists for developing this evidence base.

It could be argued that pharmacists have sufficient existing pharmaceutical knowledge to manage most deprescribing conversations with a reasonable level of confidence. The ideal model of proactive deprescribing may therefore be that the doctor initiates discussion with the patient about their medicines and the scope for discontinuation. A pharmacist then undertakes the consultation with the responsibility for developing and implementing the trial discontinuation in partnership with the patient and doctor.

Deprescribing support resources generally focus on assisting with clinical decision-making, and there is little consideration of the other barriers and enablers to implementation. It is now widely accepted that changing behaviour requires an understanding of the processes of change associated with adopting a new behaviour. ${ }^{[11]}$ This understanding can be facilitated through the use of behaviour change theory. Accordingly, researchers and policymakers should draw on behaviour change theory in addition to the literature and empirical evidence when developing strategies to facilitate proactive deprescribing.

In summary, the deprescribing of greatest interest and driving government and research agendas regarding medicines optimization is 'proactive'. Whilst it has the greatest potential for reducing drug budgets and preventing problematic polypharmacy, it is complex and requires evidence-based approaches for implementation. This provides numerous opportunities for pharmacists to play a role in initiating, implementing and supporting the process. So, whilst reactive deprescribing is routine clinical practice for pharmacists, proactive deprescribing represents exciting practice and research opportunities.

\section{References}

1. Scott IA et al. Reducing inappropriate polypharmacy: the process of deprescribing. JAMA Intern Med 2015; 175: 827-834.

2. Scott $\mathrm{S}$ et al. Deprescribing admission medication at a UK teaching hospital; a report on quantity and nature of activity. Int J Clin Pharm 2018; 1-6 https:// doi.org/10.1007/s11096-018-0673-1.

3. Pérez T et al. Prevalence of potentially inappropriate prescribing in older people in primary care and its association with hospital admission: longitudinal study. BMJ 2018; 363: k4524.

4. Anderson $\mathrm{K}$ et al. Negotiating, "Unmeasurable Harm and Benefit": perspectives of general practitioners and consultant pharmacists on deprescribing in the primary care setting. Qual Health Res 2017; 27: 1936-1947.

5. Reeve E et al. Patient barriers to and enablers of deprescribing: a systematic review. Drugs Aging 2013; 30: 793-807.

6. Anderson $\mathrm{K}$ et al. Prescriber barriers and enablers to minimising potentially inappropriate medications in adults: a systematic review and thematic synthesis. BMJ Open 2014; 4: e006544.

7. Thillainadesan $\mathrm{J}$ et al. Impact of deprescribing interventions in older hospitalised patients on prescribing and clinical outcomes: a systematic review of randomised trials. Drugs Aging [Internet] 2018;35:303-319.
8. Page AT et al. The feasibility and effect of deprescribing in older adults on mortality and health: a systematic review and meta-analysis. $\mathrm{Br} J \mathrm{Clin}$ Pharmacol 2016; 82: 583-623.

9. Farrell B et al. Deprescribing proton pump inhibitors: evidence-based clinical practice guideline. Can Fam Physician 2017; 63: 354-364.

10. Farrell B et al. Deprescribing antihyperglycemic agents in older persons: evidence-based clinical practice guideline. Can Fam Physician 2017; 63: 832-843.

11. Craig $\mathrm{P}$ et al. Developing and Evaluating Complex Interventions: The New Medical Research Council Guidance. London: British Medical Journal Publishing Group, 2008. 